\title{
The Anti-Ischemic and Anti-Anginal Properties of Statins
}

\author{
Joel A. Lardizabal • Prakash C. Deedwania
}

Published online: 24 November 2010

(C) The Author(s) 2010. This article is published with open access at Springerlink.com

\begin{abstract}
Angina pectoris resulting from myocardial ischemia afflicts half of all patients with coronary heart disease (CHD). Chronic angina remains a major public health burden despite state-of-the-art therapies, and improvement in survival from myocardial infarction and CHD has only increased its prevalence. There is growing experimental and clinical evidence pointing to the antiischemic and anti-anginal properties of statins. Some data suggest that the degree of anti-ischemic efficacy of statins may be comparable to the current standard pharmacologic and mechanical strategies. The pleiotropic effects of statins are postulated to be primarily responsible for their anti-ischemic and anti-anginal properties. These include improvement of endothelial function, enhancement of the ischemic vasodilatory response, modulation of inflammation, and protection from ischemia-reperfusion injury. The anti-ischemic effects of statins further strengthen their role as a crucial component of the optimal medical therapy for CHD.
\end{abstract}

\footnotetext{
J. A. Lardizabal

Division of Cardiology, Fresno Medical Education Program, University of California - San Francisco,

155 N. Fresno St.,

Fresno, CA 93301, USA

P. C. Deedwania

University of California - San Francisco School of Medicine,

San Francisco, CA, USA

P. C. Deedwania $(\bowtie)$

Veterans Affairs Central California Healthcare System, Division of Cardiology, VACCHS Medical Center,

2615 E Clinton Avenue,

Fresno, CA 93703, USA

e-mail: pdeedwania@fresno.ucsf.edu
}

Keywords Lipid-lowering · Statin · Cardiovascular disease $\cdot$ Coronary heart disease $\cdot$ Myocardial ischemia . Angina pectoris

\section{Introduction}

Coronary heart disease (CHD), the leading cause of death for both men and women, is highly prevalent, afflicting nearly 18 million individuals in the United States alone [1]. About half of all patients with CHD experience angina pectoris. Chronic angina is a common and a very costly public health burden. Disbursements from angina-related hospital admissions, coronary procedures, and outpatient treatment can consume more than $1 \%$ of the total health care expenditure in the United States [2]. Advances in the management of CHD have dramatically improved the survival from acute coronary events. Chronic angina is a recurrent symptom most commonly encountered by these patients [3]. Ironically, as the number of survivors of myocardial infarction (MI) increases, so does the prevalence of chronic angina.

Angina is a clinical syndrome that is commonly defined as chest discomfort and related symptoms resulting from myocardial ischemia. Global or regional myocardial ischemia occurs when there is a mismatch between myocardial oxygen supply and demand, which is most often associated with significant occlusion of one or more epicardial coronary arteries [4]. A variety of pharmacologic and mechanical strategies comprise the current armamentarium of angina therapy. The standard anti-anginal medications include the beta-blocker, calcium channel antagonist, nitrate, and piperazine classes of agents. Mechanical approaches to anti-anginal management include coronary revascularization (through either percutaneous or surgical 
methods) and non-invasive techniques (such as enhanced external counterpulsation).

There has been mounting evidence suggesting that the HMG-CoA reductase inhibitor (statin) class of cholesterollowering agents possesses anti-anginal properties to a degree of efficacy that may be equivalent to the standard pharmacologic and mechanical therapies. Understanding the expanded role of statins in anti-ischemic therapy is highly relevant, particularly in the context of recent clinical trial data that show optimal medical therapy alone is at least as effective as coronary revascularization in the initial management of patients with chronic stable angina [5•].

\section{Statins and Myocardial Ischemia}

The mortality and morbidity benefit of statins in the management of CHD is well established, both in the primary and secondary prevention settings. In high-risk individuals without prior history of CHD, statin therapy, on average, reduces the risk of MI by $27 \%$ and overall mortality by $7 \%$ [6]. In patients with known CHD, it is estimated that statin treatment reduces total mortality by $16 \%$, CHD mortality by $23 \%$, and major CHD events by $25 \%$, irrespective of baseline cholesterol levels [7].

There is ample literature verifying the favorable influence of statins on myocardial ischemia in patients with CHD. The Regression Growth Evaluation Statin Study (REGRESS), for instance, assessed the effects of pravastatin on transient myocardial ischemia using ambulatory electrocardiograpy (AECG) in nearly 800 patients with known CHD who were on routine anti-anginal therapy [8]. After 2 years of followup, the trial showed that statin therapy significantly reduced the duration of transient myocardial ischemia and the total ischemic burden by nearly half compared to baseline, whereas no improvement was seen with placebo. This beneficial effect remained even after adjusting for major ischemic risk factors, as well as revascularization procedures such as percutaneous coronary intervention (PCI) or coronary artery bypass grafting (CABG). Furthermore, statin therapy was associated with a significant $42 \%$ decrement in major adverse clinical events.

The Vascular Basis for the Treatment of Myocardial Ischemia Study randomized 300 dyslipidemic patients with stable CHD and documented ischemia on stress testing or AECG into treatment strategies of intensive lipid-lowering to a target low-density lipoprotein (LDL) of $<80 \mathrm{mg} / \mathrm{dL}$ (using high-dose atorvastatin with or without antioxidant vitamins) or moderate lipid-lowering with diet and lowdose lovastatin to a target LDL of $<130 \mathrm{mg} / \mathrm{dL}$ [9]. Ischemia evaluation using AECG and stress testing were conducted at 6 and 12 months. After 1 year of treatment, the study showed that statin therapy, using either intensive- or moderate-dosing strategies, led to significant reductions in the duration of ischemia and frequency of angina episodes. Statin treatment also resulted in significant improvement in exercise time prior to onset of ischemia. No significant differences in outcomes were seen between the intensive and moderate lipid-lowering groups despite a significantly larger lowering of LDL levels in the intensive arm. It was postulated that there may be a threshold effect of LDL lowering, below which there is no incremental improvement in ischemic parameters, and that the lipid levels achieved with either statin treatment strategies were below this threshold.

Another major clinical trial, the Study Assessing Goals in the Elderly (SAGE), compared intensive (atorvastatin $80 \mathrm{mg}$ daily) versus moderate (pravastatin $40 \mathrm{mg}$ daily) statin therapy in nearly 900 patients with CHD and at least one documented episode of myocardial ischemia on AECG [10]. After 12 months of follow-up, the trial demonstrated that statin therapy, using either intensive-dosing or moderate-dosing strategies, was associated with a significant $37 \%$ reduction in the total duration of myocardial ischemia on AECG, a benefit that was evident as early as 3 months (Fig. 1). Like that seen in the Vascular Basis Study, there was no significant difference between treatment groups in the total duration of ischemia despite a greater cholesterol reduction in those who received intensive statin therapy. However, a trend toward fewer occurences of major adverse cardiac events was also seen in intensive lipid-lowering arm. Notably, high-dose atorvastatin was associated with a significant $77 \%$ reduction in total mortality. These findings suggest that the magnitude of LDL lowering that is effective in the stabilization of

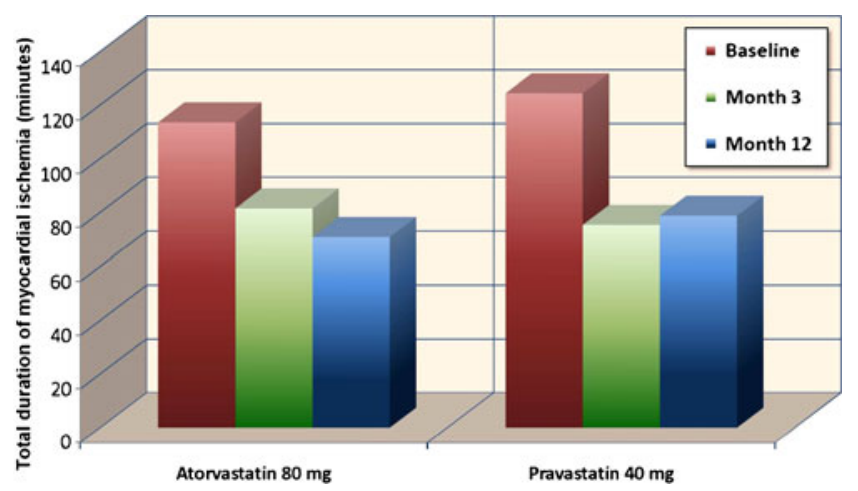

Fig. 1 Mean total duration of myocardial ischemia on 48-hour ambulatory electrocardiogram at baseline, month 3, and month 12 in 893 patients treated with either atorvastatin or pravastatin in the Study Assessing Goals in the Elderly (SAGE) trial [10]. (Adapted from Deedwania P, Stone PH, Bairey Merz CN, et al.: Effects of intensive versus moderate lipid-lowering therapy on myocardial ischemia in older patients with coronary heart disease: results of the Study Assessing Goals in the Elderly (SAGE). Circulation 2007, 115 (6):700-707; with permission) 
vulnerable plaque and the reduction of clinical events may be continuous (ie, progressively lower LDL values are associated with progressively lower cardiac event rates). When taken in context of the results of the Vascular Basis Study, it appears that the mechanisms responsible for the benefits of lipid-lowering therapy in clinical event reduction may differ from that of ischemia. It is hypothesized that improvement in ischemia is predominantly related to improvement in myocardial oxygen supply-and-demand balance, whereas acute cardiac events are more closely related to plaque disruption or rupture.

\section{The Anti-Anginal Effects of Statins}

The reduction in myocardial ischemic burden seen with statin therapy is expected to be accompanied by improvement in angina-related symptomatology. The Scandinavian Simvastatin Survival Study (4S) was one of the earliest clinical trials that demonstrated the possible anti-anginal properties of statins [11]. In this study, 4444 dyslipidemic patients with CHD were randomized to treatment with either simvastatin or placebo for 5.4 years. Post-hoc analysis of the $4 \mathrm{~S}$ trial showed a $26 \%$ reduction in new or worsening angina, as well as a $37 \%$ lowering in the incidence of coronary revascularization, with statin therapy. Simvastatin treatment significantly decreased the risk of new or worsening intermittent claudication and bruits as well.

In 69 patients with symptomatic CHD, a substudy of the REGRESS trial assessed regional myocardial perfusion and angina functional class at baseline and after 2 years of treatment with either pravastatin or placebo [12]. The study showed a significant improvement in regional myocardial perfusion in the patients who received pravastatin, whereas deterioration was seen in those assigned to placebo. The enhancement in myocardial perfusion was accompanied by significant improvement in angina functional class, and this change was seen only in patients who received statin therapy. Furthermore, the group of patients on pravastatin had fewer clinical events and had better preserved exercise capacity on stress testing compared to placebo [12].

There is evidence that suggests that the anti-ischemic efficacy of statins is comparable to that of the more established pharmacologic anti-anginal agents. The Double-blind Atorvastatin Amlodipine (DUAAL) trial enrolled over 300 patients with symptomatic CHD and compared the anti-ischemic effects of atorvastatin with that of the calcium channel blocker (CCB) amlodipine, a standard anti-anginal agent [13]. After 24 weeks of therapy, both treatment arms induced a comparable, highly significant reduction in myocardial ischemia documented with
AECG and exercise testing. The marked decrease in the frequency of angina and nitroglycerin consumption seen with the $\mathrm{CCB}$ was found to be equivalent to that of the statin, leading to the conclusion that atorvastatin was as potent an anti-ischemic agent as amlodipine.

There are also data suggesting that aggressive lipidlowering therapy is at least as effective as mechanical reperfusion strategies in low-risk patients with chronic stable angina. In the Atorvastatin versus Revascularization Treatment (AVERT) trial, nearly 350 patients with stable CHD were randomized to either statin therapy or angioplasty [14]. After 18 months of follow-up, the study showed a 35\% lower incidence of ischemic events and a significantly longer time to the first ischemic event in the group of patients who were assigned to atorvastatin therapy. Notably, the patients who underwent angioplasty had a $52 \%$ higher incidence of worsening angina compared to those who received medical therapy alone. Although significant advances in revascularization techniques have been made since the AVERT trial, findings from the more recent Adenosine Sestamibi Post-Infarction Evaluation (INSPIRE) [15] and Clinical Outcomes Utilizing Revascularization and Aggressive Drug Evaluation (COURAGE) [16] trials provide support that lipid-lowering therapy, in combination with other guideline-based medical treatments, maybe as effective in angina management as percutaneous coronary intervention (PCI) or bypass surgery in appropriate, lowerrisk patients with stable CHD.

\section{Anti-Atherosclerotic Effects of Statins}

It is believed that the anti-ischemic properties of statins are primarily the result of their favorable effects on vascular function and atherosclerosis. The prevailing theory on atherogenesis points to the central roles of LDL and inflammation in the development of atherosclerosis. The initiating step is thought to be the oxidation of LDL, which is subsequently taken up by monocytes. This complex gets deposited into a dysfunctional vascular endothelium, forming the classic foam cell. This process promotes further lipid deposition, resulting in atherosclerotic plaque formation. Progressive plaque accumulation leads to chronic vessel stenosis, whereas rupture of this atherosclerotic lesion results in acute vascular syndromes [17, 18].

The statins are the most potent lipid-lowering agents currently available, and the newer agents have been shown to reduce LDL levels by up to $55 \%$. Statins lower LDL primarily through the inhibition of the HMG-CoAreductase enzyme, which catalyzes the first committed step of cholesterol synthesis in the mevalonate pathway. Statins also decrease triglyceride levels to a lesser degree (up to $20 \%$ ), presumably through the inhibition of its synthesis in 
the liver and enhancement of lipoprotein lipase enzyme activity in the adipocytes $[19,20]$. In addition, statins also increase the levels of high-density lipoprotein (HDL), albeit modestly (up to $10 \%$ ), the precise mechanism for which remains unclear. It is postulated that the HDL-raising properties of statins result from the induction of the apolipoprotein A1 (Apo-A1) gene through the activation of peroxisome proliferator-activated receptors [21]. HDL inhibits the progression of the atherosclerotic process by facilitating the reverse transport of cholesterol from lipidladen monocytes in the vascular wall, and also by modulating the inflammatory response [17].

Clinical studies have shown that the improvement in lipid profile seen with statin therapy halts the progression, and even induces the regression, of atherosclerosis. The Measuring Effects on Intima-Media Thickness: an Evaluation of Rosuvastatin (METEOR) trial enrolled nearly 1,000 asymptomatic individuals with subclinical atherosclerosis, who were given either rosuvastatin or placebo [22]. The study found that, within a relatively short period of time, statin treatment significantly slowed the rate of increase of carotid intima-media thickness on ultrasonography, a change that was evident as early as 12 months within initiation of therapy [23]. On the other hand, in over 500 patients with known CHD, A Study to Evaluate the Effect of Rosuvastatin on Intravascular Ultrasound-Derived Coronary Atheroma Burden (the ASTEROID trial) was able to demonstrate a significant reduction in coronary atheroma volume and overall plaque burden (measured by intravascular ultrasound) with just 2 years of high-intensity statin therapy [24].

Statins are able to favorably affect the course of chronic stable angina presumably by halting or reversing the plaque buildup responsible for vessel stenosis. In clinical trials, however, the observed absolute change in percent stenosis is actually small, and the substantial reductions in clinical event rates appeared out of proportion to the documented arteriographic changes [25]. This suggests that the pathophysiologic mechanisms responsible for chronic stable angina are different from those involved in acute coronary events. Statins are thought to prevent acute, unstable coronary events through stabilization of the "vulnerable plaque". The vulnerable plaque is characterized by high lipid content, increased inflammatory activity, and thin fibrous cap, which predispose it to disruption and rupture. Statins deplete the lipid content and also inhibit LDL oxidation, inflammation, and apoptosis within the atherosclerotic plaque. These changes are accompanied by increased collagen content and expression of antiinflammatory mediators. All of these processes represent the alterations in the molecular structure of the atherosclerotic plaque that favor stability [26]. The phenomenon of "plaque stabilization" is probably the single most important basis for the consistent morbidity and mortality reduction seen in the different statin trials.

\section{Pleiotropic Anti-Ischemic Effects of Statins}

Animal studies have demonstrated that chronic statin therapy can preserve myocardial perfusion and coronary microvascular permeability independent of lipid lowering [27]. Such an effect of statins on coronary blood flow was verified in human studies that utilized myocardial perfusion imaging (MPI). One trial randomized a small number of patients with known CHD and average cholesterol levels into treatment with either pravastatin or placebo for 16 weeks, followed by a cross-over period that lasted another 16 weeks. Dypiridamole stress radionuclide MPI testing was performed at the end of each study period. Analysis of MPI data showed that patients had significantly smaller perfusion defects on dipyridamole stress testing while on statin therapy than when on placebo [28]. This improvement was seen after only a short period of treatment, suggesting that the benefit derived from statin therapy is related to functional rather than anatomic changes.

Another trial prospectively assessed the effects of statin treatment on the lipid profile and myocardial perfusion in patients with CHD and dyslipidemia during the first 6 months of pravastatin therapy. Cholesterol levels were monitored and serial radionuclide MPI were performed at baseline, at 6 weeks, and at 6 months of statin treatment. At the end of the follow-up period, half of the subjects showed significant improvement in myocardial perfusion. The direction and magnitude of changes in myocardial perfusion did not correlate with improvement in cholesterol profile, suggesting a lipid-independent phenomenon [29].

Improvement in myocardial perfusion occurs with statin therapy, even in the absence of arteriographic evidence of atherosclerotic regression [12]. In addition to the fixed atherosclerotic obstruction (endothelial dysfunction), it is likely that dynamic coronary vasoconstriction resulting from abnormal vasomotor function probably is the dominant factor contributing to episodes of myocardial ischemia. Coronary angiographic studies have demonstrated a paradoxical vasoconstrictive response to acetylcholine infusion in coronary arteries that have minimal obstructive lesions. This highlights the significance of endothelial dysfunction in coronary vascular bed where there is early-stage atherosclerosis, and such phenomenon may indeed contribute to myocardial ischemia, which is primarily related to decreased supply rather than increased demand [30]. These observations reinforce the postulation that certain pleiotropic properties of statins are responsible for their antiischemic properties (Fig. 2). 
Fig. 2 Proposed mechanisms and sites of action for the anti-anginal and anti-ischemic effects of statins in coronary heart disease

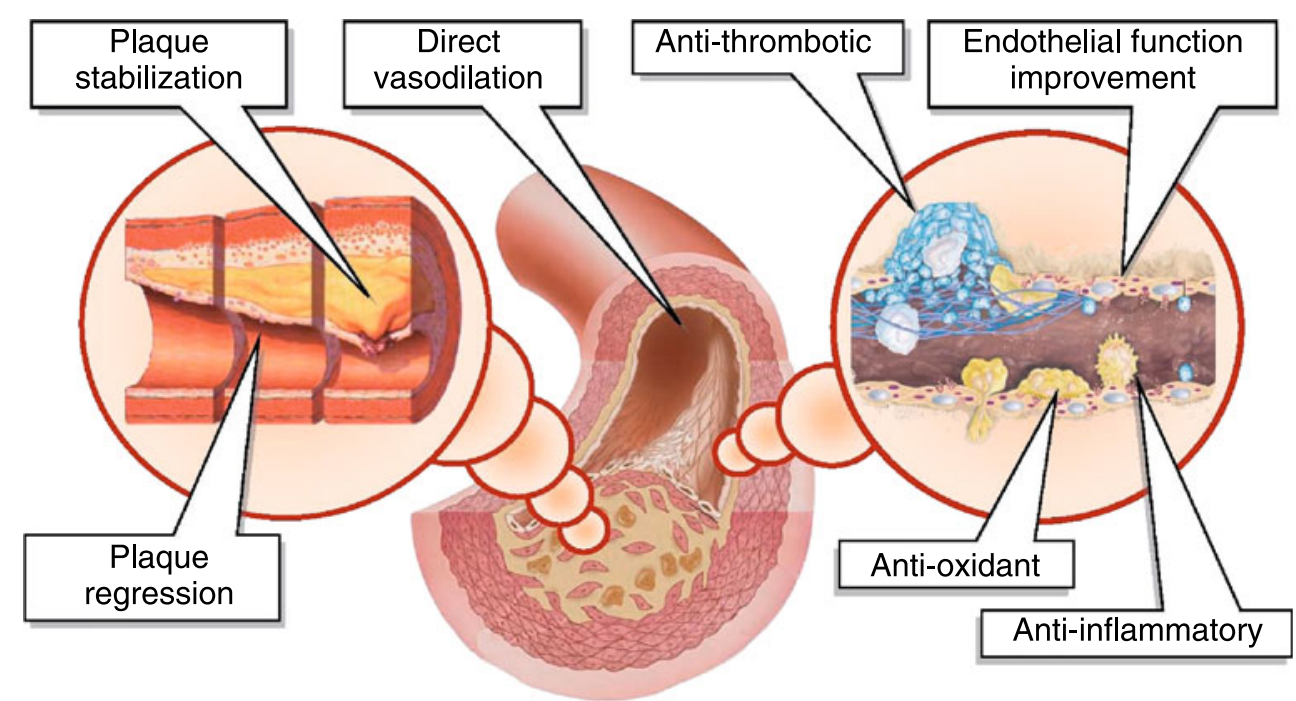

Improvement of Endothelial Function by Statins

There is ample experimental evidence pointing to the lipidindependent effects of statins on endothelial function. In healthy normocholesterolemic individuals, a single dose of cerivastatin was shown to significantly increase arterial flow-mediated dilatation (FMD), a measure of endothelial function, and this change rapidly returned to baseline $6 \mathrm{~h}$ after statin administration. There were no alterations seen in the lipid profile and in the indices of endotheliumindependent arterial dilatation, suggesting that cerivastatin may have a direct vascular effect [31]. Animal models also show that statins can augment coronary blood flow, improve cardiac contractile function, and inhibit leukocyte-endothelial cell interactions, primarily by enhancing the endothelial release of nitric oxide [32]. Nitric oxide release in endothelial cells is primarily mediated by the endothelial nitric oxide synthase (eNOS) enzyme. Experimental studies have demonstrated the ability of statins to enhance the expression, increase the activity, or prevent the inactivation of eNOS [33].

The experimental findings on the lipid-independent effects of statins on endothelial function have been corroborated by clinical data as well. A small trial randomized 60 dyslipidemic patients without CHD into treatment with either high-dose simvastatin monotherapy, combination low-dose simvastatin with ezetimibe, or placebo [34]. Lipid levels and indices of endothelial function and vascular inflammation, including C-reactive protein (CRP), Rho-associated coiled-coil containing protein kinase (ROCK), and FMD, were measured at baseline and after 4 weeks. Compared to placebo, both treatment arms significantly reduced LDL and CRP levels. Significant improvement in endothelial function (decreased ROCK activity and increased FMD) was seen in the highdose simvastatin monotherapy group but not with combi- nation low-dose simvastatin-ezetemibe therapy, despite similar degrees of LDL and CRP reductions.

The benefit of statins has also been shown in patients with cardiac syndrome X (CSX), a disorder characterized by anginal chest pain, positive exercise test, and angiographically normal coronary arteries. CSX is hypothesized to result from impairment in the normal endothelial function of the coronary microvasculature, resulting in inadequate flow reserve. Arterial FMD, as a measure of endothelial function, was assessed in small trial of 40 normolipidemic patients with CSX who were randomized into treatment with either pravastatin or placebo. After 3 months of therapy, the statin group showed significant improvement in FMD, which was accompanied by significant prolongation in exercise duration and time to significant ST-depression on stress testing [35]. The pleiotrophic effect of statins on endothelial function was presumed to be the primary mechanism responsible for these findings. These results are clinically relevant as the majority of women presenting with ischemic-type signs and symptoms do not have obstructive coronary stenoses but have impaired coronary vasoreactivity, which has the potential to limit myocardial flow and may be a predictor of major adverse outcomes [36].

\section{Anti-Inflammatory Effects of Statins and Ischemia}

Using CRP level as a marker of low-grade systemic inflammation, the Pravastatin Inflammation/CRP Evaluation (PRINCE) trial sought to evaluate the antiinflammatory effects of statins in nearly 1,200 patients with CHD and over 1,700 patients without CHD who were randomized to receive either pravastatin or placebo for 24 weeks [37]. Statin therapy was shown to significantly reduce CRP levels by up to $17 \%$, an effect that was evident as early as 12 weeks. The decrement in CRP levels did not 
correlate with co-morbid conditions, cholesterol profile, or change in LDL levels over time, implying that the antiinflammatory effects of statins might well be independent of their lipid-lowering properties.

There are also experimental data that show statins are able to suppress oxidative stress, primarily those mediated by protein kinase $\mathrm{C}$ and phospholipase $\mathrm{D}$, and that these effects may be unrelated to lipid-lowering. The protection from oxidative stress afforded by statins may prevent the migration of vascular smooth muscle cells in the arterial wall, which is an important mechanism in atherogenesis and CHD [38]. In addition, statin treatment has been demonstrated to inhibit platelet aggregation and thrombus formation in vascular territories exposed to oxidative stress [39]. Postulated mechanisms for these anti-thrombotic effects include enhancement of nitric oxide and cyclic GMP formation, which are thought to contribute further to the vasculoprotective properties of statins [40].

\section{Blunting of Ischemia-Reperfusion Injury}

Statins improve the vasodilatory response to ischemia most likely by increasing the extracellular formation of adenosine. Rosuvastatin has been shown to achieve this effect by enhancing the activity of ecto-5'-nucleotidase, the key enzyme in extracellular adenosine formation, which results in improvement of reactive hyperemia in the setting of ischemia [41]. Through this mechanism, statins may also interact synergistically with other vasodilating agents to provide protection against ischemia-reperfusion injury [42].

The clinical implications of these experimental observations can be inferred from the findings of different clinical trials on coronary revascularization. In the Novel Approaches for Preventing or Limiting Events (NAPLESII) study, nearly 700 statin-naïve patients who were scheduled to undergo elective PCI were randomized to treatment with either single high-dose atorvastatin or placebo within $24 \mathrm{~h}$ of the procedure [43]. The trial found that a single, loading-dose of statin was associated with a significant $32 \%$ reduction in the incidence of periprocedural biomarker-defined MI. This result was congruent with an earlier similar study where over 450 patients undergoing elective PCI were pre-treated with either statin or placebo at least 3 days prior to the procedure. After follow-up, the trial showed a $47 \%$ decrease in large Q-wave MI and a $27 \%$ reduction in biomarker-defined $\mathrm{MI}$ in the group who received statins [44].

Near-immediate cardioprotective effects of statins have also been demonstrated in patients undergoing surgical revascularization. One trial randomized $200 \mathrm{CHD}$ patients scheduled for coronary artery bypass grafting (CABG) to 1-week preoperative treatment with either rosuvastatin or placebo. On post-operative follow-up, the study found significantly higher levels of cardiac biomarkers and CRP in those who received placebo. Myocardial damage was seen more frequently in the high-risk patients, but the incidence was significantly less in those who were given rosuvastatin [45].

\section{Conclusions}

Improved survival from MI and CHD has led to an increase in the number of patients afflicted with chronic angina. Despite the current state-of-the-art pharmacologic and mechanical therapies, chronic angina remains a major public health burden. Evidence is mounting on the anti-ischemic and antianginal efficacy of statins in both experimental and clinical settings. The pleiotropic effects of statins may be primarily responsible for their anti-ischemic and anti-anginal properties. These pleiotropic effects include improvement of endothelial function, enhancement of the ischemic vasodilatory response, modulation of inflammation, and protection from ischemiareperfusion injury. The anti-ischemic effects of statins further strengthen their role as a crucial component of the optimal medical therapy for $\mathrm{CHD}$, and they have been shown to be as effective as coronary revascularization in the initial management of patients with chronic stable angina. The question of whether or not statins are as potent as the standard classes of anti-anginal drugs, or as effective in improving ischemia as certain mechanical strategies, may very well be considered moot because statin therapy is recommended for all patients with CHD. However, the knowledge of their anti-ischemic properties may help reinforce the logic behind treating all our CHD patients with statins.

Disclosure Joel A. Lardizabal reports no potential conflict of interest relevant to this article. Prakash C. Deedwania reports no potential conflict of interest relevant to this article.

Open Access This article is distributed under the terms of the Creative Commons Attribution Noncommercial License which permits any noncommercial use, distribution, and reproduction in any medium, provided the original author(s) and source are credited.

\section{References}

Papers of particular interest, published recently, have been highlighted as:

- Of importance

1. American Heart Association Statistics Committee and Stroke Statistics Subcommittee. Executive Summary: Heart Disease and Stroke Statistics-2010 Update: A Report From the American Heart Association. Circulation. 2010 Feb 23;121(7):948-954. 
2. Stewart S, Murphy NF, Walker A, et al. The current cost of angina pectoris to the National Health Service in the UK. Heart. 2003;89 (8):848-53.

3. Deedwania PC, Carbajal EV, Bobba VR. Trials and tribulations associated with angina and traditional therapeutic approaches. Clin Cardiol. 2007;30(2 Suppl 1):I16-24.

4. In: The AHA guidelines and scientific statements handbook. Ed. by V. Fuster. (C) 2009 American Heart Association.

5. BARI 2D Study Group. A randomized trial of therapies for type 2 diabetes and coronary artery disease. N Engl J Med. 2009;360 (24):2503-15. This study showed that optimal medical therapy is comparable to a strategy of prompt revascularization in the initial management of diabetic patients with chronic stable angina.

6. Mills EJ, Rachlis B, Wu P, Devereaux PJ, Arora P, Perri D. Primary prevention of cardiovascular mortality and events with statin treatments: a network meta-analysis involving more than 65,000 patients. J Am Coll Cardiol. 2008;52(22):1769-81.

7. Wilt TJ, Bloomfield HE, MacDonald R, et al. Effectiveness of statin therapy in adults with coronary heart disease. Arch Intern Med. 2004;164(13):1427-36.

8. van Boven AJ,Jukema JW, Zwinderman AH, et al. Reduction of Transient Myocardial Ischemia With Pravastatin in Addition to the Conventional Treatment in Patients With Angina Pectoris. Circ 1996;94:1503-1505.

9. Stone PH, Lloyd-Jones DM, Kinlay S, et al. Effect of intensive lipid lowering, with or without antioxidant vitamins, compared with moderate lipid lowering on myocardial ischemia in patients with stable coronary artery disease: the Vascular Basis for the Treatment of Myocardial Ischemia Study. Circulation. 2005 ;111 (14):1747-55.

10. Deedwania P, Stone PH, Bairey Merz CN, et al. Effects of intensive versus moderate lipid-lowering therapy on myocardial ischemia in older patients with coronary heart disease: results of the Study Assessing Goals in the Elderly (SAGE). Circ 2007;115 (6):700-7.

11. Pedersen TR, Kjekshus J, Pyörälä K, et al. Effect of simvastatin on ischemic signs and symptoms in the Scandinavian simvastatin survival study (4S). Am J Cardiol. 1998;81(3):333-5.

12. Aengevaeren WR, Uijen GJ, Jukema JW, et al. Functional evaluation of lipid-lowering therapy by pravastatin in the Regression Growth Evaluation Statin Study (REGRESS). Circulation. 1997;96(2):429-35.

13. Deanfield JE, Selleir P, Thaulow E, et al. Potent anti-ischaemic effects of statins in chronic stable angina: incremental benefit beyond lipid lowering? Eur Hrt J. 2010 [Epub].

14. Pitt B, Waters D, Brown WV, et al. Aggressive lipid-lowering therapy compared with angioplasty in stable coronary artery disease. Atorvastatin versus Revascularization Treatment Investigators. N Engl J Med. 1999;341(2):70-6.

15. The INSPIRE Investigators. An initial strategy of intensive medical therapy is comparable to that of coronary revascularization for suppression of scintigraphic ischemia in high-risk but stable survivors of acute myocardial infarction. J Am Coll Cardiol. 2006;48(12):2458-67.

16. COURAGE Trial Research Group. Optimal medical therapy with or without PCI for stable coronary disease. N Engl J Med. 2007;356(15):1503-16.

17. Fuster V, Moreno PR, Fayad ZA, Corti R, Badimon JJ. Atherothrombosis and high-risk plaque: part I: evolving concepts. J Am Coll Cardiol. 2005;46(6):937-54.

18. Navab M, Ananthramaiah GM, Reddy ST, et al. The oxidation hypothesis of atherogenesis: the role of oxidized phospholipids and HDL. J Lipid Res. 2004;45(6):993-1007.

19. Saiki A, Murano T, Watanabe F, et al. Pitavastatin enhanced lipoprotein lipase expression in 3T3-L1 preadipocytes. J Atheroscler Thromb. 2005;12(3):163-8.
20. Jones PH, Davidson MH, Stein EA, et al. Comparison of the efficacy and safety of rosuvastatin versus atorvastatin, simvastatin, and pravastatin across doses (STELLAR* Trial). Am J Cardiol. 2003;92(2):152-60.

21. Yano M, Matsumura T, Senokuchi T, et al. Statins activate peroxisome proliferator-activated receptor gamma through extracellular signalregulated kinase $1 / 2$ and p38 mitogen-activated protein kinasedependent cyclooxygenase-2 expression in macrophages. Circ Res. 2007;100(10):1442-51.

22. Crouse JR 3rd, Raichlen JS, Riley WA, et al. Effect of rosuvastatin on progression of carotid intima-media thickness in low-risk individuals with subclinical atherosclerosis: the METEOR Trial. JAMA. 2007;297(12):1344-53.

23. Bots ML, Palmer MK, Dogan S, et al. Intensive lipid lowering may reduce progression of carotid atherosclerosis within 12 months of treatment: the METEOR study. J Intern Med. 2009;265(6):698-707.

24. Nissen SE, Nicholls SJ, Sipahi I, et al. Effect of very highintensity statin therapy on regression of coronary atherosclerosis: the ASTEROID trial. JAMA. 2006;295(13):1556-65.

25. Brown BG, Zhao XQ, Sacco DE, Albers JJ. Lipid lowering and plaque regression. New insights into prevention of plaque disruption and clinical events in coronary disease. Circulation. 1993;87(6):1781-91.

26. Crisby M, Nordin-Fredriksson G, Shah PK, et al. Pravastatin treatment increases collagen content and decreases lipid content, inflammation, metalloproteinases, and cell death in human carotid plaques: implications for plaque stabilization. Circulation. 2001;103(7):926-33.

27. Bonetti PO, Wilson SH, Rodriguez-Porcel M, et al. Simvastatin preserves myocardial perfusion and coronary microvascular permeability in experimental hypercholesterolemia independent of lipid lowering. J Am Coll Cardiol. 2002;40(3):546-54.

28. Mostaza JM, Gomez MV, Gallardo F, et al. Cholesterol reduction improves myocardial perfusion abnormalities in patients with coronary artery disease and average cholesterol levels. J Am Coll Cardiol. 2000;35(1):76-82.

29. Schwartz RG, Pearson TA, Kalaria VG, et al. Prospective serial evaluation of myocardial perfusion and lipids during the first six months of pravastatin therapy: coronary artery disease regression single photon emission computed tomography monitoring trial. J Am Coll Cardiol. 2003;42(4):600-10.

30. McLenachan JM, Williams JK, Fish RD, et al. Loss of flowmediated endothelium-dependent dilation occurs early in the development of atherosclerosis. Circulation. 1991;84(3):1273-8.

31. Omori H, Nagashima H, Tsurumi Y, et al. Direct in vivo evidence of a vascular statin: a single dose of cerivastatin rapidly increases vascular endothelial responsiveness in healthy normocholesterolaemic subjects. Br J Clin Pharmacol. 2002;54(4):395-9.

32. Lefer AM, Campbell B, Shin YK, et al. Simvastatin preserves the ischemic-reperfused myocardium in normocholesterolemic rat hearts. Circulation. 1999;100(2):178-84.

33. Feron O, Dessy C, Desager JP, et al. Hydroxy-methylglutarylcoenzyme A reductase inhibition promotes endothelial nitric oxide synthase activation through a decrease in caveolin abundance. Circulation. 2001;103(1):113-8.

34. Liu PY, Liu YW, Lin LJ, et al. Evidence for statin pleiotropy in humans: differential effects of statins and ezetimibe on rhoassociated coiled-coil containing protein kinase activity, endothelial function, and inflammation. Circulation. 2009;119(1):131-8.

35. Kayikcioglu M, Payzin S, Yavuzgil O, et al. Benefits of statin treatment in cardiac syndrome-X. Eur Heart J. 2003;24(22):1999 2005.

36. Pepine CJ, Anderson RD, Sharaf BL, et al. Coronary microvascular reactivity to adenosine predicts adverse outcome in women evaluated for suspected ischemia results from the National Heart, Lung and 
Blood Institute WISE (Women's Ischemia Syndrome Evaluation) study. J Am Coll Cardiol. 2010;55(25):2825-32.

37. Albert MA, Danielson E, Rifai N, et al. Effect of statin therapy on C-reactive protein levels: the pravastatin inflammation/CRP evaluation (PRINCE): a randomized trial and cohort study. JAMA. 2001;286(1):64-70.

38. Yasunari K, Maeda K, Minami M, et al. HMG-CoA reductase inhibitors prevent migration of human coronary smooth muscle cells through suppression of increase in oxidative stress. Arterioscler Thromb Vasc Biol. 2001;21(6):937-42.

39. Gaddam V, Li DY, Mehta JL. Anti-thrombotic effects of atorvastatin - an effect unrelated to lipid lowering. J Cardiovasc Pharmacol Ther. 2002;7(4):247-53.

40. Chou TC, Lin YF, Wu WC, et al. Enhanced nitric oxide and cyclic GMP formation plays a role in the anti-platelet activity of simvastatin. Br J Pharmacol. 2008 Mar;153(6):1281-7.

41. Meijer P, Wouters CW, van den Broek PH, et al. Upregulation of Ecto-5'-Nucleotidase by Rosuvastatin Increases the Vasodilator
Response to Ischemia. Hypertension. 2010;Aug 2 [Epub ahead of print].

42. Meijer P, Oyen WJ, Dekker D, et al. Rosuvastatin increases extracellular adenosine formation in humans in vivo: a new perspective on cardiovascular protection. Arterioscler Thromb Vasc Biol. 2009;29(6):963-8.

43. Briguori C, Visconti G, Focaccio A, et al. Novel approaches for preventing or limiting events (Naples) II trial: impact of a single high loading dose of atorvastatin on periprocedural myocardial infarction. J Am Coll Cardiol. 2009;54(23):215763.

44. Briguori C, Colombo A, Airoldi F, et al. Statin administration before percutaneous coronary intervention: impact on periprocedural myocardial infarction. Eur Heart J. 2004;25(20):1822-8.

45. Mannacio VA, Iorio D, De Amicis V, et al. Effect of rosuvastatin pretreatment on myocardial damage after coronary surgery: a randomized trial. J Thorac Cardiovasc Surg. 2008;136 (6):1541-8. 